\title{
Functionalized Polyethyleneimine-gold Nanoparticles-Porphyrin Nanocomposite for Electrochemical Glucose Biosensing
}

\author{
Shumei $\mathrm{Gu}^{1 \ddagger}$, Kefeng Ma ${ }^{1 \ddagger}$, Jinming Kong ${ }^{1, *}$, Khalid A. Al-Ghanim ${ }^{2}$, Shahid Mahboob ${ }^{2}$, \\ Ying Liul and Xueji Zhang ${ }^{3, *}$ \\ ${ }^{1}$ School of Environmental and Biological Engineering, Nanjing University of Science and \\ Technology, Nanjing 210094, P. R. China \\ ${ }^{2}$ Department of Zoology, College of Science, King Saud University, P.O. Box 2455, Riyadh-11451, \\ Saudi Arabia \\ ${ }^{3}$ Chemistry Department, College of Arts and Sciences, University of South Florida, East Fowler Ave, \\ Tampa, Florida 33620-4202, United States \\ ${ }^{\ddagger}$ These authors contributed equally to this work. \\ *E-mail: j.kong@njust.edu.cn, xueji@usf.edu
}

doi: $10.20964 / 2017.06 .85$

Received: 18 March 2017 / Accepted: 24 April 2017 / Published: 12 May 2017

In this work, a novel polyethyleneimine-gold nanoparticles-porphyrin (PEI-AuNPs-Fe ${ }^{\mathrm{III}} \mathrm{MPIX}$ ) nanocomposite was constructed by a simple method to achieve excellent electrochemical properties. Firstly, AuNPs were reduced and stabilized by PEI, and then Fe ${ }^{\mathrm{III}}$ MPIX was conjugated to this scaffold through amidation reaction. After that, the nanocomposite was functionalized by 4mercaptophenylboronic acid (MPBA) through Au-S bond for the determination of glucose. By virtue of high affinity of MPBA for 1, 2-diols of glucose, electrochemical activity of Fe ${ }^{\text {III }}$ MPIX and the synergistic effect of PEI-coated AuNPs, this biosensor displayed good sensitivity and selectivity for glucose. The electrochemical response was linearly related to glucose concentration in the range from 10 to $350 \mu \mathrm{M}\left(R^{2}=0.9911\right)$. A low detection limit of $2.16 \mu \mathrm{M}(\mathrm{S} / \mathrm{N}=3)$ and a high sensitivity of 13.88 $\mu \mathrm{A} \mathrm{mM}{ }^{-1} \mathrm{~cm}^{-2}$ was obtained. In addition, this biosensor exhibited excellent anti-interference ability against common interferents such as ascorbic acid (AA), dopamine (DA), alanine (Ala) and proline (Pro), and held satisfactory analytical performance for the determination of glucose in human serum samples, indicating promising practical applications in bioanalysis and clinical diagnosis.

Keywords: Porphyrin; Gold nanoparticle; Polyethyleneimine; 4-Mercaptophenylboronic acid; Glucose sensor

$\underline{\text { FULL TEXT }}$ 
(C) 2017 The Authors. Published by ESG (www.electrochemsci.org). This article is an open access article distributed under the terms and conditions of the Creative Commons Attribution license (http://creativecommons.org/licenses/by/4.0/). 